\title{
Creation operators and algebraic Bethe ansatz for the elliptic quantum group $E_{\tau, \eta}\left(s O_{3}\right)$
}

\author{
Nenand Manojlovićc and Zoltán Nagy ${ }^{\dagger}$ \\ Departamento de Matemática \\ FCT, Campus de Gambelas \\ Universidade do Algarve \\ 8005-139 Faro, Portugal
}

\begin{abstract}
We define the elliptic quantum group $E_{\tau, \eta}\left(\mathrm{so}_{3}\right)$ and the transfer matrix corresponding to its simplest highest weight representation. We use Bethe anstaz method to construct the creation operators as polynomials of the Lax matrix elements expressed through a recurrence relation. We give common eigenvectors and eigenvalues of the family of commuting transfer matrices.
\end{abstract}

\section{Introduction}

In this article, we report new results on the application of algebraic Bethe ansatz to the elliptic (or dynamical) quantum group $E_{\tau, \eta}\left(s o_{3}\right)$. The elliptic quantum group is the algebraic structure associated to elliptic solutions of the star-triangle relation. This equation appears in interaction-round-a-face models in statistical mechanics. As it was shown by Felder [4], this structure is also related to Knizhnik-ZamolodchikovBernard equation of conformal field theory on tori. Moreover, in a different direction, to each solution of the (see [9]) star-triangle relation a dynamical $R$-matrix can be associated. This $R$-matrix, in turn, will define an algebra similar to quantum groups appearing in the quantum inverse scattering method (QISM), it is actually a quasi-Hopf deformation of the more familiar quantum group structure [10. Despite all the differences, this new structure preserves a prominent feature of quantum groups: a tensor product of representations can be defined.

The adjective dynamical refers to the fact that the $R$-matrix appearing in these structures contains a parameter which in the classical limit will be interpreted as the position coordinate on the phase space of a classical system and the resulting classical $r$-matrix will depend

*e-mail address: nmanoj@ualg.pt

†e-mail address: znagy@ualg.pt 
on it. In the quantum setting, apart from the appearance of this extra parameter the Yang-Baxter equation (YBE) is also deformed. At the technical level, the main difference between usual quantum groups and the one we are about to describe lies not so much in the elliptic nature of the appearing functions as rather in the introduction of the extra "dynamical" parameter and the corresponding deformation of the YBE.

In QISM, the physically interesting quantity is the transfer matrix. The hamiltonian of the model and other observables are derived from it. The knowledge of its spectrum is thus essential. Different kinds of methods under the federating name of Bethe ansatz have been developed to calculate the eigenvalues of the transfer matrix [3, 11, 12]. The question whether the algebraic Bethe ansatz (ABA) technique can be applied to transfer matrices appearing in the context of dynamical quantum groups has received an affirmative answer from Felder and Varchenko [7, 5]. They showed how to implement ABA for the elliptic quantum group $E_{\tau, \eta}\left(s l_{2}\right)$, they also showed its applications to IRF models and Lamé equation. Later, for the $E_{\tau, \eta}\left(s l_{n}\right)$ elliptic quantum group the nested Bethe ansatz method was used [2, 8] and a relation to Ruijsenaars-Schneider [8] and quantum Calogero-Moser Hamiltonians was established [1.

In the first section of this paper we introduce the basic definitions of dynamical $R$-matrix, Yang-Baxter equation, representations, operator algebra and commuting transfer matrices. We define elements $\Phi_{n}$ in the operator algebra which have the necessary symmetry properties to be the creation operators of the corresponding Bethe states. As it turns out, the creation operators are not simple functions of the Lax matrix entries, unlike in [5], but they are complicated polynomials of three generators $A_{1}(u), B_{1}(u), B_{2}(u)$ in the elliptic operator algebra. We give the recurrence relation which defines the creation operators. Moreover, we fully implement the algebraic Bethe ansatz on the simplest example of highest weight module. That is we calculate the action of the transfer matrix on the Bethe vectors and from the vanishing of the unwanted terms we derive the Bethe equations. We also give the explicit formulas for the corresponding eigenvalues.

\section{Representations of $E_{\tau, \eta}\left({S O_{3}}_{3}\right)$ and transfer matrices}

\subsection{Definitions}

Let us first recall the basic definitions which will enter our construction. First, we fix two complex numbers $\tau, \eta$ such that $\operatorname{Im}(\tau)>0$. The central object in this paper is the $R$-matrix $R(q, u)$ which depends on two arguments $q, u \in \mathbb{C}$ : the first one is referred to as the dynamical parameter, the second one is called the spectral parameter. The ele- 
ments of the $R$-matrix are written in terms of Jacobi's theta function:

$$
\vartheta(u)=-\sum_{j \in \mathbb{Z}} \exp \left(\pi i\left(j+\frac{1}{2}\right)^{2}+2 \pi i\left(j+\frac{1}{2}\right)\left(u+\frac{1}{2}\right)\right)
$$

This function has two essential properties. It is quasiperiodic:

$$
\vartheta(u+1)=-\vartheta(u) ; \quad \vartheta(u+\tau)=-e^{-i \tau-2 i u} \vartheta(u)
$$

and it verifies the identity:

$$
\begin{aligned}
& \vartheta(u+x) \vartheta(u-x) \vartheta(v+y) \vartheta(v-y)=\vartheta(u+y) \vartheta(u-y) \vartheta(v+x) \vartheta(v-x)+ \\
& \vartheta(u+v) \vartheta(u-v) \vartheta(x+y) \vartheta(x-y)
\end{aligned}
$$

The entries of the $R$-matrix are written in terms of the following functions.

$$
\begin{aligned}
g(u) & =\frac{\vartheta(u-\eta) \vartheta(u-2 \eta)}{\vartheta(\eta) \vartheta(2 \eta)} \\
\alpha\left(q_{1}, q_{2}, u\right) & =\frac{\vartheta(\eta-u) \vartheta\left(q_{12}-u\right)}{\vartheta(\eta) \vartheta\left(q_{12}\right)} \\
\beta\left(q_{1}, q_{2}, u\right) & =\frac{\vartheta(\eta-u) \vartheta(u) \vartheta\left(q_{12}-2 \eta\right)}{\vartheta(-2 \eta) \vartheta(\eta) \vartheta\left(q_{12}\right)} \\
\varepsilon(q, u) & =\frac{\vartheta(\eta+u) \vartheta(2 \eta-u)}{\vartheta(\eta) \vartheta(2 \eta)}-\frac{\vartheta(u) \vartheta(\eta-u)}{\vartheta(\eta) \vartheta(2 \eta)}\left(\frac{\vartheta(q+\eta) \vartheta(q-2 \eta)}{\vartheta(q-\eta) \vartheta(q)}+\frac{\vartheta(q-\eta) \vartheta(q+2 \eta)}{\vartheta(q+\eta) \vartheta(q)}\right) \\
\gamma\left(q_{1}, q_{2}, u\right) & =\frac{\vartheta(u) \vartheta\left(q_{1}+q_{2}-\eta-u\right) \vartheta\left(q_{1}-2 \eta\right) \vartheta\left(q_{2}+\eta\right)}{\vartheta(\eta) \vartheta\left(q_{1}+q_{2}-2 \eta\right) \vartheta\left(q_{1}+\eta\right) \vartheta\left(q_{2}\right)} \\
\delta(q, u) & =\frac{\vartheta(u-q) \vartheta(u-q+\eta)}{\vartheta(q) \vartheta(q-\eta)}
\end{aligned}
$$

The $R$-matrix itself will act on the tensor product $V \otimes V$ where $V$ is a three-dimensional complex vector space with the standard basis $\left\{e_{1}, e_{2}, e_{3}\right\}$. The matrix units $E_{i j}$ are defined in the usual way: $E_{i j} e_{k}=$ $\delta_{j k} e_{i}$. We will also need the following diagonal matrix later on: $h=$ $E_{11}-E_{33}$.

Now we are ready to write the explicit form of the $R$-matrix.

$$
\begin{aligned}
R(q, u) & =g(u) E_{11} \otimes E_{11}+g(u) E_{33} \otimes E_{33}+\varepsilon(q, u) E_{22} \otimes E_{22} \\
& +\alpha(\eta, q, u) E_{12} \otimes E_{21}+\alpha(q, \eta, u) E_{21} \otimes E_{12}+\alpha(-q, \eta, u) E_{23} \otimes E_{32} \\
& +\alpha(\eta,-q, u) E_{32} \otimes E_{23} \\
& +\beta(\eta, q, u) E_{11} \otimes E_{22}+\beta(q, \eta, u) E_{22} \otimes E_{11}+\beta(-q, \eta, u) E_{22} \otimes E_{33} \\
& +\beta(\eta,-q, u) E_{33} \otimes E_{22} \\
& +\gamma(-q, q, u) E_{11} \otimes E_{33}+\gamma(-q, \eta, u) E_{12} \otimes E_{32}-\gamma(\eta, q, u) E_{21} \otimes E_{23} \\
& +\gamma(q,-q, u) E_{33} \otimes E_{11}+\gamma(q, \eta, u) E_{32} \otimes E_{12}-\gamma(\eta,-q, u) E_{23} \otimes E_{21} \\
& +\delta(q, u) E_{31} \otimes E_{13}+\delta(-q, u) E_{13} \otimes E_{31}
\end{aligned}
$$

This $R$-matrix also enjoys the unitarity property:

$$
R_{12}(q, u) R_{21}(q,-u)=g(u) g(-u) \mathbb{1}
$$


and it is of zero weight:

$$
\left[h \otimes \mathbb{1}+\mathbb{1} \otimes h, R_{12}(q, u)\right]=0 \quad(h \in \mathfrak{h})
$$

The $R$-matrix also obeys the dynamical quantum Yang-Baxter equation (DYBE) in $\operatorname{End}(V \otimes V \otimes V)$ :

$$
\begin{aligned}
& R_{12}\left(q-2 \eta h_{3}, u_{12}\right) R_{13}\left(q, u_{1}\right) R_{23}\left(q-2 \eta h_{1}, u_{2}\right)= \\
& R_{23}\left(q, u_{2}\right) R_{13}\left(q-2 \eta h_{2}, u_{1}\right) R_{12}\left(q, u_{12}\right)
\end{aligned}
$$

where the "dynamical shift" notation has the usual meaning:

$$
R_{12}\left(q-2 \eta h_{3}, u\right) \cdot v_{1} \otimes v_{2} \otimes v_{3}=\left(R_{12}(q-2 \eta \lambda, u) v_{1} \otimes v_{2}\right) \otimes v_{3}
$$

whenever $h v_{3}=\lambda v_{3}$. Shifts on other spaces are defined in an analogous manner.

Let us also describe a more intuitive way of looking at this shift. Define first the shift operator acting on functions of the dynamical parameter:

$$
\exp \left(2 \eta \partial_{q}\right) f(q)=f(q+2 \eta) \exp \left(2 \eta \partial_{q}\right)
$$

Then equation (21) can also be written in the following form:

$$
R_{12}\left(q-2 \eta h_{3}, u\right)=\exp \left(-2 \eta h_{3} \partial_{q}\right) R_{12}(q, u) \exp \left(2 \eta h_{3} \partial_{q}\right)
$$

in the sequel we will use whichever definition is the fittest for the particular point in our calculation.

\subsection{Representation; operator algebra}

Now we describe the notion of representation of (or module over) $E_{\tau, \eta}\left(s o_{3}\right) . \quad$ It is a pair $(\mathcal{L}(q, u), W)$ where $W$ is a diagonalizable $\mathfrak{h}$ module, that is, $W$ is a direct sum of the weight subspaces $W=$ $\oplus \in \mathbb{C} W[]$ and $\mathcal{L}(q, u)$ is an operator in $\operatorname{End}(V \otimes W)$ obeying:

$$
\begin{aligned}
& R_{12}\left(q-2 \eta h_{3}, u_{12}\right) \mathcal{L}_{13}\left(q, u_{1}\right) \mathcal{L}_{23}\left(q-2 \eta h_{1}, u_{2}\right)= \\
& \mathcal{L}_{23}\left(q, u_{2}\right) \mathcal{L}_{13}\left(q-2 \eta h_{2}, u_{1}\right) R_{12}\left(q, u_{12}\right)
\end{aligned}
$$

$\mathcal{L}(q, u)$ is also of zero weight

$$
\left[h_{V} \otimes \mathbb{1}+\mathbb{1} \otimes h_{W}, \mathcal{L}_{V, W}(q, u)\right]=0 \quad(h \in \mathfrak{h})
$$

where the subscripts remind the careful reader that in this formula $h$ might act in a different way on spaces $W$ and $V$.

An example is given immediately by $W=V$ and $\mathcal{L}(q, u)=R(q, u-$ $z)$ which is called the fundamental representation with evaluation point $z$ and is denoted by $V(z)$. A tensor product of representations can also be defined which corresponds to the existence of a coproduct-like structure at the abstract algebraic level. Let $(\mathcal{L}(q, u), X)$ and $\left(\mathcal{L}^{\prime}(q, u), Y\right)$ be two $E_{\tau, \eta}\left(s o_{3}\right)$ modules, then $\mathcal{L}_{1 X}\left(q-2 \eta h_{Y}, u\right) \mathcal{L}_{1 Y}(q, u), X \otimes Y$ is a representation of $E_{\tau, \eta}\left(s o_{3}\right)$ on $X \otimes Y$ endowed, of course, with the tensor product $\mathfrak{h}$-module structure. 
The operator $\mathcal{L}$ is reminiscent of the quantum Lax matrix in the FRT formulation of the quantum inverse scattering method, although it obeys a different exchange relation, therefore we will also call it a Lax matrix. This allows us to view the $\mathcal{L}$ as a matrix with operator-valued entries.

Inspired by that interpretation, for any module over $E_{\tau, \eta}\left(s_{3}\right)$ we define the corresponding operator algebra of finite difference operators. Let us take an arbitrary representation $\mathcal{L}(q, u) \in \operatorname{End}(V \otimes W)$. The elements of the operator algebra corresponding to this representation will act on the space $\operatorname{Fun}(W)$ of meromorphic functions of $q$ with values in $W$. Namely let $L \in \operatorname{End}(V \otimes \operatorname{Fun}(W))$ be the operator defined as:

$$
L(u)=\left(\begin{array}{lll}
A_{1}(u) & B_{1}(u) & B_{2}(u) \\
C_{1}(u) & A_{2}(u) & B_{3}(u) \\
C_{2}(u) & C_{3}(u) & A_{3}(u)
\end{array}\right)=\mathcal{L}(q, u) e^{-2 \eta h \partial_{q}}
$$

We can view it as a matrix with entries in $\operatorname{End}(\operatorname{Fun}(W))$ : It follows from equation (3) that $L$ verifies:

$R_{12}\left(q-2 \eta h, u_{12}\right) L_{1 W}\left(q, u_{1}\right) L_{2 W}\left(q, u_{2}\right)=L_{2 W}\left(q, u_{2}\right) L_{1 W}\left(q, u_{1}\right) \tilde{R}_{12}\left(q, u_{12}\right)$

with $\tilde{R}_{12}(q, u):=\exp \left(2 \eta\left(h_{1}+h_{2}\right) \partial_{q}\right) R_{12}(q, u) \exp \left(-2 \eta\left(h_{1}+h_{2}\right) \partial_{q}\right)$

The zero weight condition on $L$ yields the relations:

$$
\begin{array}{r}
{\left[h, A_{i}\right]=0 ; \quad\left[h, B_{j}\right]=-B_{j} \quad(j=1,3),\left[h, B_{2}\right]=-2 B_{2}} \\
{\left[h, C_{j}\right]=C_{j} \quad(j=1,3),\left[h, C_{2}\right]=2 C_{2}}
\end{array}
$$

so $B_{i}$ 's act as lowering and $C_{i}$ 's as raising operators. From the definition (3) one can derive the action of the operator algebra generators on functions:

$$
\begin{array}{r}
A_{1}(u) f(q)=f(q-2 \eta) A_{1}(u) ; B_{1}(u) f(q)=f(q) B_{1}(u) \\
B_{2}(u) f(q)=f(q+2 \eta) B_{2}(u)
\end{array}
$$

and analogously for the other generators. We display here those commutation relations which are necessary for the construction of the Bethe vectors, the remaining ones can be extracted from (4).

$$
\begin{aligned}
B_{1}\left(u_{1}\right) B_{1}\left(u_{2}\right) & =\omega_{21}\left(B_{1}\left(u_{2}\right) B_{1}\left(u_{1}\right)-\frac{1}{y_{21}(q)} B_{2}\left(u_{2}\right) A_{1}\left(u_{1}\right)\right)+\frac{1}{y_{12}(q)} B_{2}\left(u_{1}\right) A_{1}\left(u_{2}\right) \\
A_{1}\left(u_{1}\right) B_{1}\left(u_{2}\right) & =z_{21}(q) B_{1}\left(u_{2}\right) A_{1}\left(u_{1}\right)-\frac{\alpha_{21}(\eta, q)}{\beta_{21}(q, \eta)} B_{1}\left(u_{1}\right) A_{1}\left(u_{2}\right) \\
A_{1}\left(u_{1}\right) B_{2}\left(u_{2}\right) & =\frac{1}{\gamma_{21}(q,-q)}\left(g_{21} B_{2}\left(u_{2}\right) A_{1}\left(u_{2}\right)+\gamma_{21}(\eta,-q) B_{1}\left(u_{1}\right) B_{1}\left(u_{2}\right)-\delta_{21}(-q) B_{2}\left(u_{1}\right) A_{1}\left(u_{1}\right)\right) \\
B_{1}\left(u_{2}\right) B_{2}\left(u_{1}\right) & =\frac{1}{g_{21}}\left(\beta_{21}(-q, \eta) B_{2}\left(u_{1}\right) B_{1}\left(u_{2}\right)+\alpha_{21}(\eta,-q) B_{1}\left(u_{1}\right) B_{2}\left(u_{2}\right)\right) \\
B_{2}\left(u_{2}\right) B_{1}\left(u_{1}\right) & =\frac{1}{g_{21}}\left(\beta_{21}(\eta,-q) B_{1}\left(u_{1}\right) B_{2}\left(u_{2}\right)+\alpha_{21}(-q, \eta) B_{2}\left(u_{1}\right) B_{1}\left(u_{2}\right)\right)
\end{aligned}
$$


where

$$
\begin{aligned}
\omega(q, u) & =\frac{g(u) \gamma(q,-q, u)}{\varepsilon(q, u) \gamma(q,-q, u)+\gamma(q, \eta, u) \gamma(\eta,-q, u)} \\
y(q, u) & =\frac{\gamma(q,-q, u)}{\gamma(q, \eta, u)} \\
z(q, u) & =\frac{g(u)}{\beta(q, \eta, u)}
\end{aligned}
$$

and as usual

$$
y_{12}(q)=y\left(q, u_{1}-u_{2}\right) \text { etc. }
$$

Furthermore, the function $\omega(q, u)$ is actually independent of $q$, a property which will prove important later on, and takes the following simple form:

$$
\omega(u)=\frac{\vartheta(u+\eta) \vartheta(u-2 \eta)}{\vartheta(u-\eta) \vartheta(u+2 \eta)}
$$

This function also verifies the following property:

$$
\omega(u) \omega(-u)=1 \text {. }
$$

Finally the following theorem shows how to associate a family of commuting quantities to a representation of the elliptic quantum group

Theorem 2.1. Let $W$ be a representation of $E_{\tau, \eta}\left(s_{3}\right)$. Then the transfer matrix defined by $t(u)=\operatorname{Tr} \tilde{L}(u) \in \operatorname{End}(\operatorname{Fun}(W))$ preserves the subspace $\operatorname{Fun}(W)[0]$ of functions with values in the zero weight subspace of $W$. When restricted to this subspace, they commute at different values of the spectral parameter:

$$
[t(u), t(v)]=0
$$

Proof. The proof is analogous to references [6, 1]

\section{$3 \quad$ Bethe ansatz}

In this section we fix a highest weight module $W=V\left(z_{1}\right) \otimes \ldots \otimes V\left(z_{n}\right)$. The vector $|0\rangle=e_{1} \otimes \ldots \otimes e_{1} \in \operatorname{Fun}(W)$ is a highest weight vector of weight $n$ of this module, and every highest weight vector can be written in the form $|\Omega\rangle=f(q)|0\rangle$ with a non-zero meromorphic function $f$. We have indeed:

$$
C_{i}(u)|\Omega\rangle=0 \quad(i=1,2,3)
$$

showing that $|\Omega\rangle$ is a highest weight vector.

$$
\begin{array}{r}
A_{1}(u)|\Omega\rangle=a_{1}(u) \frac{f(q-2 \eta)}{f(q)}|\Omega\rangle \\
A_{2}(u)|\Omega\rangle=a_{2}(q, u)|\Omega\rangle \quad A_{3}(u)|\Omega\rangle=a_{3}(q, u) \frac{f(q+2 \eta)}{f(q)}|\Omega\rangle
\end{array}
$$


with the eigenvalues:

$$
\begin{aligned}
a_{1}(u) & =\prod_{i=1}^{n} \frac{\vartheta\left(u-z_{i}-\eta\right) \vartheta\left(u-z_{i}-2 \eta\right)}{\vartheta(\eta) \vartheta(2 \eta)} \\
a_{2}(q, u) & =\frac{\vartheta(q-2 \eta n-\eta)}{\vartheta(q-\eta)} \prod_{i=1}^{n} \frac{\vartheta\left(u-z_{i}-\eta\right) \vartheta\left(u-z_{i}\right)}{\vartheta(\eta) \vartheta(2 \eta)} \\
a_{3}(q, u) & =\frac{\vartheta(q-2 \eta n) \vartheta(q-2 \eta n+\eta)}{\vartheta(q+\eta) \vartheta(q)} \prod_{i=1}^{n} \frac{\vartheta\left(u-z_{i}\right) \vartheta\left(u-z_{i}+\eta\right)}{\vartheta(\eta) \vartheta(2 \eta)}
\end{aligned}
$$

Notice that $a_{1}$ is independent of $q$. It is easy to see, that the zero weight subspace $W[0]$ is nontrivial for this module.

In this situation we cannot look for the common eigenvectors of $t(u)$ in the form $\Phi\left(u_{1}, \ldots, u_{n}\right)=B_{1}\left(u_{1}\right) \ldots B_{1}\left(u_{n}\right)|\Omega\rangle$ since $B_{1}(u) B_{1}(v) \neq$ $B_{1}(v) B_{1}(u)$ and the resulting Bethe vector would not be symmetric under the interchange of the parameters $u_{i}$.

Instead, we should be inspired by Tarasov's implementation of the algebraic Bethe ansatz to the Izergin-Korepin model [15]. In that case the $R$-matrix is nondynamical, but has nonzero entries at the same positions as the dynamical $R$-matrix considered here. The comparison suggests that the Bethe operator will contain $B_{1}\left(u_{1}\right) \ldots B_{1}\left(u_{n}\right)$ with coefficient 1 but will also have a "correction" to that expressed in terms of $B_{2}(u)$ and $A_{1}(u)$. It also suggests that the symmetry under the interchange of spectral parameters is replaced by the property:

$\Phi_{n}\left(u_{1}, \ldots, u_{n}\right)=\zeta_{i+1, i} \Phi_{n}\left(u_{1}, \ldots, u_{i-1}, u_{i+1}, u_{i}, u_{i+2}, \ldots, u_{n}\right) \quad(i=1, \ldots, n-1)$

with a function $\zeta$ to be determined. In the sequel we give the Bethe creation operator in a recurrence form and describe its generalized symmetry property.

Definition 3.1. Let $\Phi_{n}$ be defined be the recurrence relation for $n \geq 0$ :

$$
\begin{aligned}
& \Phi_{n}\left(u_{1}, \ldots, u_{n}\right)=B_{1}\left(u_{1}\right) \Phi_{n-1}\left(u_{2}, \ldots, u_{n}\right) \\
& -\sum_{j=2}^{n} \frac{\prod_{k=2}^{j-1} \omega_{j k}}{y_{1 j}(q)} \prod_{\substack{k=2 \\
k \neq j}}^{n} z_{k j}(q+2 \eta) B_{2}\left(u_{1}\right) \Phi_{n-2}\left(u_{2}, \ldots, \widehat{u_{j}}, \ldots, u_{n}\right) A_{1}\left(u_{j}\right)
\end{aligned}
$$

where $\Phi_{0}=1 ; \Phi_{1}\left(u_{1}\right)=B_{1}\left(u_{1}\right)$ and the parameter under the hat is omitted.

It may be useful to give explicitly the first three creation operators.

$$
\begin{aligned}
& \Phi_{1}\left(u_{1}\right)=B_{1}\left(u_{1}\right) \\
& \Phi_{2}\left(u_{1}, u_{2}\right)=B_{1}\left(u_{1}\right) B_{1}\left(u_{2}\right)-\frac{1}{y_{12}(q)} B_{2}\left(u_{1}\right) A_{1}\left(u_{2}\right) \\
& \Phi_{3}\left(u_{1}, u_{2}, u_{3}\right)=B_{1}\left(u_{1}\right) B_{1}\left(u_{2}\right) B_{1}\left(u_{3}\right)-\frac{1}{y_{23}(q)} B_{1}\left(u_{1}\right) B_{2}\left(u_{2}\right) A_{1}\left(u_{3}\right) \\
& -\frac{z_{32}(q+2 \eta)}{y_{12}(q)} B_{2}\left(u_{1}\right) B_{(}\left(u_{3}\right) A_{1}\left(u_{2}\right)-\frac{\omega_{32} z_{23}(q+2 \eta)}{y_{13}(q)} B_{2}\left(u_{1}\right) B_{1}\left(u_{2}\right) A_{1}\left(u_{3}\right)
\end{aligned}
$$


The Bethe vector is then not completely symmetric under the interchange of two neighboring spectral parameters but verifies the following property instead:

$$
\begin{aligned}
& \Phi_{2}\left(u_{1}, u_{2}\right)=\omega_{21} \Phi_{2}\left(u_{2}, u_{1}\right) \\
& \Phi_{3}\left(u_{1}, u_{2}, u_{3}\right)=\omega_{21} \Phi_{3}\left(u_{2}, u_{1}, u_{3}\right)=\omega_{32} \Phi_{3}\left(u_{1}, u_{3}, u_{2}\right)
\end{aligned}
$$

For general $n$ we prove the following theorem.

Theorem 3.1. $\Phi_{n}$ verifies the following symmetry property:

$\Phi_{n}\left(u_{1}, \ldots, u_{n}\right)=\omega_{i+1, i} \Phi_{n}\left(u_{1}, \ldots, u_{i-1}, u_{i+1}, u_{i}, u_{i+2}, \ldots, u_{n}\right) \quad(i=1,2, \ldots, n-1)$.

Proof. For the proof we refer to [13].

The next step in the application of the Bethe ansatz scheme is the calculation of the action of the transfer matrix on the Bethe vector. This will yield three kinds of terms. The first part (usually called wanted terms in the literature) will tell us the eigenvalue of the transfer matrix, the second part (called unwanted terms) must be annihilated by a careful choice of the spectral parameters $u_{i}$ in $\Phi_{n}\left(u_{1}, \ldots, u_{n}\right)$; the vanishing of these unwanted terms is ensured if the $u_{i}$ are solutions to the so called Bethe equations. The third part contains terms ending with a raising operator acting on the pseudovacuum and thus vanishes.

The action of $A_{1}(u)$ on $\Phi_{n}$ is given by

$$
\begin{aligned}
A_{1}(u) \Phi_{n}= & \prod_{k=1}^{n} z_{k u}(q) \Phi_{n} A_{1}(u)+ \\
& \sum_{j=1}^{n} D_{j} \prod_{k=1}^{j-1} \omega_{j k} B_{1}(u) \Phi_{n-1}\left(u_{1}, \hat{u}_{j}, u_{n}\right) A_{1}\left(u_{j}\right)+ \\
& \sum_{l<j}^{n} E_{l j} \prod_{k=1}^{l-1} \omega_{l k} \prod_{\substack{k=1 \\
k \neq l}}^{j-1} \omega_{j k} B_{2}(u) \Phi_{n-2}\left(u_{1}, \hat{u}_{l}, \hat{u}_{j}, u_{n}\right) A_{1}\left(u_{l}\right) A_{1}\left(u_{j}\right)
\end{aligned}
$$

To calculate the first coefficients we expand $\Phi_{n}$ with the help of the recurrence relation, then use the commutation relations to push $A_{1}\left(u_{1}\right)$ to the right. This yields:

$$
\begin{aligned}
D_{1} & =-\frac{\alpha_{1 u}(\eta, q)}{\beta_{1 u}(q, \eta)} \prod_{k=2}^{n} z_{k 1}(q) \\
E_{12} & =\left(\frac{\delta_{1 u}(-q)}{\gamma_{1 u}(q,-q) y_{12}(q-2 \eta)}+\frac{z_{1 u}(q) \alpha_{2 u}(\eta, q) \omega_{u 1}}{\beta_{2 u}(q, \eta) y_{u 1}(q)}\right) \prod_{k=3}^{n} z_{k 1}(q+2 \eta) z_{k 2}(q)
\end{aligned}
$$

The direct calculation of the remaining coefficients is less straightforward. However, the symmetry of the left hand side of (6) implies that $D_{j}$ for $j \geq 1$ can be obtained by substitution $u_{1} \rightsquigarrow u_{j}$ in $D_{1}$ and $E_{l j}$ by the substitution $u_{1} \rightsquigarrow u_{l}, u_{2} \rightsquigarrow u_{j}$ 
The action of $A_{2}(u)$ and $A_{3}(u)$ on $\Phi_{n}$ will yield also terms ending in $C_{i}(u)$ 's.

The action of $A_{2}(u)$ on $\Phi_{n}$ will have the following structure.

$$
\begin{aligned}
A_{2}(u) \Phi_{n}= & \prod_{k=1}^{n} \frac{z_{u k}(q-2 \eta(k-1))}{\omega_{u k}} \Phi_{n} A_{2}(u)+ \\
& \sum_{j=1}^{n} F_{j}^{(1)} \prod_{k=1}^{j-1} \omega_{j k} B_{1}(u) \Phi_{n-1}\left(u_{1}, \hat{u}_{j}, u_{n}\right) A_{2}\left(u_{j}\right)+ \\
& \sum_{j=1}^{n} F_{j}^{(2)} \prod_{k=1}^{j-1} \omega_{j k} B_{3}(u) \Phi_{n-1}\left(u_{1}, \hat{u}_{j}, u_{n}\right) A_{1}\left(u_{j}\right)+ \\
& \sum_{l<j}^{n} G_{l j}^{(1)} \prod_{k=1}^{l-1} \omega_{l k} \prod_{\substack{k=1 \\
k \neq l}}^{j-1} \omega_{j k} B_{2}(u) \Phi_{n-2}\left(u_{1}, \hat{u}_{l}, \hat{u}_{j}, u_{n}\right) A_{1}\left(u_{l}\right) A_{2}\left(u_{j}\right)+ \\
& \sum_{l<j}^{n} G_{l j}^{(2)} \prod_{k=1}^{l-1} \omega_{l k}^{j-1} \prod_{\substack{k=1 \\
k \neq l}}^{j} \omega_{j k} B_{2}(u) \Phi_{n-2}\left(u_{1}, \hat{u}_{l}, \hat{u}_{j}, u_{n}\right) A_{1}\left(u_{j}\right) A_{2}\left(u_{l}\right)+ \\
& \sum_{l<j}^{n} G_{l j}^{(3)} \prod_{k=1}^{l-1} \omega_{l k}^{j-1} \prod_{\substack{k=1 \\
k \neq l}} \omega_{j k} B_{2}(u) \Phi_{n-2}\left(u_{1}, \hat{u}_{l}, \hat{u_{j}}, u_{n}\right) A_{2}\left(u_{l}\right) A_{1}\left(u_{j}\right)+
\end{aligned}
$$

terms ending in $C$

We give the coefficients $D_{1}^{(k)}$ and $E_{12}^{(k)}$, the remaining ones are obtained by the same substitution as for $A_{1}(u)$

$$
\begin{aligned}
F_{1}^{(1)} & =-\frac{\alpha_{u 1}(q-2 \eta, \eta)}{\beta_{u 1}(q, \eta)} \prod_{k=2}^{n} \frac{z_{1 k}(q-2 \eta(k-1))}{\omega_{1 k}} \\
F_{1}^{(2)} & =\frac{1}{y_{u 1}(q)} \prod_{k=2}^{n} z_{k 1}(q+2 \eta) \\
G_{12}^{(1)} & =\frac{1}{y_{u 1}(q)}\left(\frac{z_{u 1}(q) \alpha_{u 2}(q-2 \eta, \eta)}{\beta_{u 2}(q-2 \eta, \eta)}-\frac{\alpha_{u 1}(q, \eta) \alpha_{12}(q-2 \eta, \eta)}{\beta_{u 1}(q, \eta) \beta_{12}(q-2 \eta, \eta)}\right) \prod_{k=3}^{n} \frac{z_{k 1}(q+2 \eta) z_{2 k}(q-2 \eta(k-1))}{\omega_{2 k}} \\
G_{12}^{(2)} & =\frac{\alpha_{u 1}(q, \eta) \alpha_{12}(q-2 \eta, \eta)}{\beta_{u 1}(q, \eta) y_{u 1}(q) \beta_{12}(q-2 \eta, \eta)} \prod_{k=3}^{n} \frac{z_{k 2}(q+2 \eta) z_{1 k}(q-2 \eta(k-1))}{\omega_{1 k}} \\
G_{12}^{(3)} & =\frac{\alpha_{u 1}(q, \eta)}{\beta_{u 1}(\eta,-q)}\left(\frac{z_{u 1}(q)}{\omega_{u 1} y_{u 2}(q)}-\frac{\alpha_{u 1}(\eta,-q)}{y_{12}(q) \beta_{u 1}(q, \eta)}\right) \prod_{k=3}^{n} \frac{z_{k 2}(q+2 \eta) z_{1 k}(q-2 \eta(k-2))}{\omega_{1 k}}
\end{aligned}
$$

It is instructing to give explicitly the expression of $F_{l}^{(1)}$

$$
F_{l}^{(1)}=-\frac{\alpha_{u l}(q-2 \eta, \eta)}{\beta_{u l}(q, \eta)} \times \frac{\vartheta(q-3 \eta)}{\vartheta(q-2 \eta n-\eta)} \prod_{\substack{k=1 \\ k \neq l}}^{n} \frac{\vartheta\left(u_{l k}-2 \eta\right)}{\vartheta\left(u_{l k}\right) \omega_{l k}}
$$


The action of $A_{3}(u)$ on the Bethe vector is somewhat simpler.

$$
\begin{aligned}
A_{3}(u) \Phi_{n}= & \prod_{k=1}^{n} \frac{\beta_{u k}(\eta,-q)}{\gamma_{u k}(q-2 \eta(k-1),-)} \Phi_{n} A_{3}(u)+ \\
& \sum_{j=1}^{n} H_{j} \prod_{k=1}^{j-1} \omega_{j k} B_{3}(u) \Phi_{n-1}\left(u_{1}, \hat{u}_{j}, u_{n}\right) A_{2}\left(u_{j}\right)+ \\
& \sum_{l<j}^{n} I_{l j} \prod_{k=1}^{l-1} \omega_{l k} \prod_{\substack{k=1 \\
k \neq l}}^{j-1} \omega_{j k} B_{2}(u) \Phi_{n-2}\left(u_{1}, \hat{u}_{l}, \hat{u}_{j}, u_{n}\right) A_{2}\left(u_{l}\right) A_{2}\left(u_{j}\right)+ \\
& \text { terms ending in } C
\end{aligned}
$$

where to save space used the notation $\gamma_{u k}(x,-)=\gamma_{u k}(x,-x)$. We give the coefficients $H_{1}$ and $I_{12}$, the rest can be obtained by the substitution of the spectral parameters as before.

$$
\begin{aligned}
& H_{1}=-\frac{1}{y_{u 1}(q)} \prod_{k=2} \frac{z_{1 k}(q-2 \eta(k-2))}{\omega_{1 k}} \\
& I_{12}=\frac{1}{\gamma_{u 2}(q,-q)}\left(\frac{\delta_{u 2}(q)}{y_{12}(q-2 \eta)}-\frac{\alpha_{u 1}(q, \eta)}{y_{u 2}(q-2 \eta)}\right) \prod_{k=3} \frac{z_{2 u}(q-2 \eta(k-2)) z_{1 u}(q-2 \eta(k-2))}{\omega_{1 k} \omega_{2 k}}
\end{aligned}
$$

The next step is to find conditions for the cancelation of the unwanted terms. We write the action of the transfer matrix in the following form:

$$
\begin{aligned}
& t(u) \Phi_{n}|\Omega\rangle=\Lambda \Phi_{n}|\Omega\rangle+ \\
& \sum_{j=1}^{n} K_{j}^{(1)} \prod_{k=1}^{j-1} \omega_{j k} B_{1}(u) \Phi_{n}\left(u_{1}, \hat{u}_{j}, u_{n}\right)+ \\
& \sum_{l<j}^{n} K_{l j}^{(2)} \prod_{k=1}^{l-1} \omega_{l k} \prod_{\substack{k=1 \\
k \neq l}}^{j-1} \omega_{j k} B_{2}(u) \Phi_{n}\left(u_{1}, \hat{u}_{l}, \hat{u}_{j}, u_{n}\right)+ \\
& \sum_{j=1}^{n} K_{j}^{(3)} \prod_{k=1}^{j-1} \omega_{j k} B_{3}(u) \Phi_{n}\left(u_{1}, \hat{u}_{j}, u_{n}\right)
\end{aligned}
$$

The general form of the eigenvalue is written as

$$
\begin{aligned}
\Lambda\left(u,\left\{u_{j}\right\}\right)= & \prod_{k=1}^{n} z_{k u}(q) \times a_{1}(q, u) \frac{f(q-2 \eta)}{f(q)}+\prod_{k=1}^{n} \frac{z_{u k}(q-2 \eta(k-1))}{\omega_{u k}} \times a_{2}(q, u)+ \\
& \prod_{k=1}^{n} \frac{\beta_{u k}(\eta,-q)}{\gamma_{u k}(q-2 \eta(k-1),-)} \times a_{3}(q, u) \frac{f(q+2 \eta)}{f(q)} .
\end{aligned}
$$

The condition of cancelation is then $K_{j}^{(1)}=K_{j}^{(3)}=0$ for $1 \leq j$ and $K_{l j}^{(2)}=0$ for $1 \leq l \leq j$ with the additional requirement that these three different kinds of condition should in fact lead to the same set of $n$ nonlinear Bethe equations fixing the $n$ parameters of $\Phi_{n}$. 
Let us first consider the coefficient $K_{1}^{(1)}$ :

$$
K_{1}^{(1)}=D_{1} a_{1}\left(u_{1}\right) \frac{f(q-2 \eta)}{f(q)}+F_{1}^{(1)} a_{2}\left(q, u_{1}\right)
$$

The condition $K_{1}^{(1)}=0$ is then equivalent to:

$$
\frac{a_{1}\left(u_{1}\right)}{a_{2}\left(q, u_{1}\right)}=\frac{f(q)}{f(q-2 \eta)} \prod_{k=2}^{n} \frac{\vartheta\left(u_{k 1}+\eta\right)}{\vartheta\left(u_{k 1}-\eta\right)} \times \frac{\vartheta(q-3 \eta)^{n}}{\vartheta(q-\eta)^{n-1} \vartheta(q-2 \eta n-\eta)}
$$

Now it remains to check that the remaining two conditions lead to the same Bethe equations. The condition

$$
0=K_{1}^{(3)}=F_{1}^{(2)} a_{1}\left(u_{1}\right) \frac{f(q)}{f(q+2 \eta)}+H_{1} a_{2}(q+2 \eta)
$$

yields the same Bethe equation as in (7) thanks to the identity (from the unitarity condition (1)):

$$
\frac{\alpha(\eta, q, u)}{\beta(q, \eta, u)}=-\frac{\alpha(q, \eta,-u)}{\beta(q, \eta,-u)}
$$

Finally, the cancelation of $K_{12}^{(2)}$ leads also to the same Bethe equation (77) thanks to the following identity:

$$
\begin{aligned}
0= & \left(\frac{\delta_{1 u}(-q)}{\gamma_{1 u}(q,-q) y_{12}(q-2 \eta)}+\frac{z_{1 u}(q) \alpha_{2 u}(\eta, q) \omega_{u 1}}{\beta_{2 u}(q, \eta) y_{u 1}(q)}\right) \times \vartheta(q-3 \eta)^{2}+ \\
& \left(\frac{\delta_{u 1}(q)}{\gamma_{u 1}(q,-q) y_{12}(q-2 \eta)}-\frac{\alpha_{u 1}(q, \eta)}{\gamma_{u 1}(q,-q) y_{u 2}(q-2 \eta)}\right) \times \vartheta(q-3 \eta)^{2}+ \\
& \frac{1}{y_{u 1}(q)}\left(\frac{z_{u 1}(q) \alpha_{u 2}(q-2 \eta, \eta)}{\beta_{u 2}(q-2 \eta, \eta)}-\frac{\alpha_{u 1}(q, \eta) \alpha_{12}(q-2 \eta, \eta)}{\beta_{u 1}(q, \eta) \beta_{12}(q-2 \eta, \eta)}\right) \times \frac{\vartheta\left(u_{21}+\eta\right) \vartheta(q-5 \eta) \vartheta(q-\eta)}{\vartheta\left(u_{21}-\eta\right)}+ \\
& \frac{\alpha_{u 1}(q, \eta) \alpha_{12}(q+2 \eta, \eta)}{\beta_{u 1}(q, \eta) y_{u 1}(q) \beta_{12}(q-2 \eta, \eta)} \times \frac{\vartheta\left(u_{12}+\eta\right) \vartheta(q-5 \eta) \vartheta(q-\eta)}{\vartheta\left(u_{12}-\eta\right)}+ \\
& \frac{\alpha_{u 1}(q, \eta)}{\beta_{u 1}(\eta,-q)}\left(\frac{z_{u 1}(q)}{\omega_{u 1} y_{u 2}(q)}-\frac{\alpha_{u 1}(\eta,-q)}{\beta_{u 1}(q, \eta) y_{12}(q)}\right) \times \frac{\vartheta\left(u_{12}+\eta\right) \vartheta(q-3 \eta) \vartheta(q-\eta)^{2}}{\vartheta\left(u_{12}-\eta\right) \vartheta(q+\eta)}
\end{aligned}
$$

Now we fix $f(q)$ so as to ensure that the Bethe equation (hence its solutions) do not depend on $q$. This can be achieved by choosing $f(q)=e^{c q} \vartheta(q-\eta)^{n}$, where $c$ is an arbitrary constant.

The simultaneous vanishing of $K_{1}^{(1)}, K_{1}^{(3)}$ and $K_{12}^{(2)}$ then leads to the condition:

$$
\prod_{k=1}^{n} \frac{\vartheta\left(u_{1}-z_{k}-2 \eta\right)}{\vartheta\left(u_{1}-z_{k}\right)}=e^{2 c \eta} \times \prod_{k=2}^{n} \frac{\vartheta\left(u_{1}-u_{k}-\eta\right)}{\vartheta\left(u_{1}-u_{k}+\eta\right)}
$$

Once again, the symmetry property of the Bethe vector $\Phi_{n}$ allows us to derive easily the conditions for the remaining $u_{j}$ 's by a simple substitution of the spectral parameters. Thus we obtain the set of Bethe equations:

$$
\prod_{k=1}^{n} \frac{\vartheta\left(u_{j}-z_{k}-2 \eta\right)}{\vartheta\left(u_{j}-z_{k}\right)}=e^{2 c \eta} \times \prod_{\substack{k=1 \\ k \neq j}}^{n} \frac{\vartheta\left(u_{j}-u_{k}-\eta\right)}{\vartheta\left(u_{j}-u_{k}+\eta\right)} \quad(j=1, \ldots, n)
$$


Since $f(q)$ is now fixed, we can write the explicit form of the eigenvalues of the transfer matrix on the module $W=V\left(z_{1}\right) \otimes \ldots \otimes V\left(z_{n}\right)$ as a $q$-independent function of the solutions of the Bethe equations :

$$
\begin{aligned}
\Lambda\left(u,\left\{u_{j}\right\}\right)= & e^{-2 \eta c} \prod_{k=1}^{n} \frac{\vartheta\left(u-z_{k}-\eta\right) \vartheta\left(u-z_{k}-2 \eta\right) \vartheta\left(u-u_{k}+2 \eta\right)}{\vartheta(\eta) \vartheta(2 \eta) \vartheta\left(u-u_{k}\right)}+ \\
& +\prod_{k=1}^{n} \frac{\vartheta\left(u-z_{k}-\eta\right) \vartheta\left(u-z_{k}\right) \vartheta\left(u-u_{k}-2 \eta\right)}{\vartheta(\eta) \vartheta(2 \eta) \vartheta\left(u-u_{k}\right)}+ \\
& +e^{2 \eta c} \prod_{k=1}^{n} \frac{\vartheta\left(u-z_{k}\right) \vartheta\left(u-z_{k}+\eta\right) \vartheta\left(u-u_{k}-\eta\right)}{\vartheta(\eta) \vartheta(2 \eta) \vartheta\left(u-u_{k}+\eta\right)} .
\end{aligned}
$$

\section{Conclusions}

We showed in this paper that the algebraic Bethe ansatz method can be applied to the elliptic quantum group $E_{\tau, \eta}\left(s o_{3}\right)$. Similarly to Tarasov's implementation of the algebraic Bethe ansatz to the Izergin- Korepin model, the creation operators for the Bethe vectors are not simple products of the Lax matrix entries but are constructed through a recurrence relation. This analogy comes from the fact that the Izergin-Korepin $R$-matrix, although nondynamical, has nonzero entries at the same positions as the elliptic dynamical $R$-matrix considered here. For the simplest highest weight module available we gave the Bethe vectors, and derived the Bethe equations as well as the eigenvalues of the transfer matrix. Detailed proofs of these results will be published elsewhere [14.

\section{Acknowledgments}

We wish to thank Petr Petrovich Kulish for illuminating discussions. This work was supported by the project POCI/MAT/58452/2004, in addition to that Z. Nagy benefited from the FCT grant SFRH/BPD/25310/2005 and N. Manojlović acknowledges additional support from SFRH/BSAB/619/2006. This manuscript was completed at the Centre for Mathematical Science of the City University, London, we are grateful for their kind hospitality.

\section{References}

[1] Avan J., Babelon O, Billey E., The Gervais-Neveu-Felder equation and the quantum Calogero-Moser system, Comm. Math. Phys. 178 (1996), 281-300

[2] Billey E., Algebraic nested Bethe ansatz for the elliptic Ruijsenaars model, math.QA $/ 9806068$

[3] Faddeev L. D., How algebraic Bethe ansatz works for integrable models, in Quantum symmetries/Symétries quantiques, Proceedings of the "Les Houches summer school, Session LXIV", 1 
August-8 September 1995, Les Houches, France, Editors A. Connes, K. Gawedzki and J. Zinn-Justin, (North-Holland, Amsterdam, 1998), 149-219

[4] Felder G., Conformal field theory and integrable systems associated to elliptic curves, hep-th/9407154

[5] Felder G., Varchenko A., Algebraic Bethe ansatz for the elliptic quantum group $E_{\tau, \eta}\left(s l_{2}\right)$, Nucl. Phys. B 480 (1996), 485-503, q-alg/9605024

[6] Felder G., Varchenko A., Elliptic quantum groups and Ruijsenaars models, J. Stat. Phys. 89 (1997), 963-980, q-alg/9704005

[7] Felder G., Varchenko A., On representations of the elliptic quantum group $E_{\tau, \eta}\left(s l_{2}\right)$, Comm. Math. Phys. 181, (1996), 741-761

[8] Hou B. Y., Sasaki R., Yang W.-L., Algebraic Bethe ansatz for the elliptic quantum group $E_{\tau, \eta}\left(s l_{n}\right)$ and its applications, Nucl. Phys. $B$ 663, 467-486 (2003)

[9] Jimbo M., Miwa T., Okado M., Solvable lattice models related to the vector representation of Classical Simple Lie Algebras, Comm. Math. Phys., 116, 507-525 (1988)

[10] Jimbo M., Konno H., Odake S., Shiaraishi J., Quasi-Hopf twistors for elliptic quantum groups, q-alg/9712029

[11] Korepin V. E., Boguliubov N. M., Izergin A. G., Quantum Inverse Scattering Method and Correlation Functions, Cambridge Monograph on Mathematical Physics, Cambridge University Press, 1993

[12] Kulish P. P., Sklyanin E. K., Quantum spectral transform method. Recent developments, in Lecture Notes in Physics, Editors J. Hietarinta, C. Montonen, Springer, New York, 1982, Vol. 15b, 61119

[13] Manojlović N., Nagy Z., Construction of the Bethe state for the $E_{\tau, \eta}\left(\mathrm{so}_{3}\right)$ elliptic quantum group, to appear in Symmetry, Integrability and Geometry: Methods and Applications, Vol. 2 (2006)

[14] Manojlović N., Nagy Z., Algebraic Bethe ansatz for the elliptic quantum group $E_{\tau, \eta}\left(s o_{3}\right)$, in preparation

[15] Tarasov V. O., Algebraic Bethe ansatz for the Izergin-Korepin R-matrix, Theor. Math. Phys., 76, 793-803 (1988) 